\title{
Effectiveness of nano-calcium phosphate paste on sensitivity during and after bleaching: a randomized clinical trial
}

\section{Alessandro Dourado LOGUERCIO(a) Lidia Yileng TAY(b) \\ Daniel Rodrigo HERRERA(c) \\ Jose BAUER(d) \\ Alessandra REIS(a)}

(a) Universidade Estadual de Ponta Grossa - UEPG, School of Dentistry, Department of Restorative Dentistry, Ponta Grossa, PR, Brazil.

(b) Universidad Peruana Cayetano Heredia - UPCH, Department of Restorative Dentistry, Distrito de San Isidro, Lima, Peru.

(c) University of Campinas - Unicamp, School of Dentistry of Piracicaba, Department of Restorative Dentistry, Piracicaba, SP, Brazil.

(d) Universidade Federal do Maranhão - UFMA, School of Dentistry, Department of Dentistry I, São Luis, MA, Brazil.

Declaration of Interests: The authors certify that they have no commercial or associative interest that represents a conflict of interest in connection with the manuscript.

\section{Corresponding Author:}

Alessandro Dourado Loguercio

E-mail: aloguercio@hotmail.com

DOI: 10.1590/1807-3107BOR-2015.vol29.0099

Submitted: Oct 02, 2014

Accepted for publication: Apr 23, 2015

Last revision: Jul 02, 2015
Abstract: The study aimed to evaluate the effectiveness of in-office bleaching and associated tooth sensitivity on application of nano-calcium phosphate paste as desensitizing agent. Bleaching was performed with $35 \%$ hydrogen peroxide gel in 40 patients who were randomly divided into placebo and nano-calcium phosphate paste groups. Bleaching efficacy (BE) was evaluated using a value-oriented Vita shade guide. Tooth sensitivity was recorded using a numeric rating scale (0-4) during bleaching and up to $48 \mathrm{~h}$ after each session. The primary outcome of absolute risk of tooth sensitivity was compared using the Fisher's exact test $(\alpha=0.05)$. The intensity of tooth sensitivity and the efficacy of in-office bleaching were also statistically evaluated. No significant differences in absolute risk and intensity of tooth sensitivity were detected between the groups $(p=1.0$ and $p=0.53$, respectively). BE was also found to be similar between the groups $(p=0.67)$. Although the use of a nano-calcium phosphate paste associated with fluoride and potassium nitrate did not influence the whitening outcome, but it also did not reduce bleaching-induced tooth sensitivity.

Keywords: Dentin Desensitizing Agents; Calcium Phosphates; Tooth Bleaching Agents; Hydrogen Peroxide; Dentin Sensitivity.

\section{Introduction}

The clinical effectiveness of at-home and in-office bleaching under the supervision of a dentist has been extensively documented. ${ }^{1}$ While in-office bleaching uses high concentrations of active hydrogen peroxide, the at-home protocol uses a lower concentration of active hydrogen peroxide. The main advantage of in-office bleaching is that the effects can be attained in a shorter period of time. However, this bleaching protocol has a very high risk of tooth sensitivity, which may occasionally be severe. ${ }^{1,2}$

Bleaching-induced tooth sensitivity has been associated with microscopic surface defects and sub-surface pores in the enamel, ${ }^{3}$ with consequent reduction in enamel microhardness. ${ }^{4,5}$ This is because of the low $\mathrm{pH}$ of the in-office bleaching gels available in the market. ${ }^{6}$ It has been theorized that these defects allow rapid diffusion of the whitening agent into the pulp, resulting in inflammatory reaction ${ }^{7}$ and bleaching-induced tooth sensitivity. ${ }^{2}$ The association between low $\mathrm{pH}$ bleaching gels and risk of tooth sensitivity is still unclear. 
However, in theory, a product that encourages repair of these microscopic defects can reduce or at least minimize tooth sensitivity by diminishing the diffusion of hydrogen peroxide into the pulp. Consequently, manufacturers added different compounds such as fluoride, potassium nitrate, and different calcium phosphate salts to bleaching products. Fluoride and potassium nitrate have been frequently used to manage tooth sensitivity with clinical success. ${ }^{8,910,11}$

The incorporation of calcium phosphate salts in bleaching gels has been shown to prevent loss of minerals and reduce loss of enamel microhardness and bleaching-induced tooth sensitivity. ${ }^{4,5} \mathrm{~A}$ low risk of tooth sensitivity was reported for a calcium-containing in-office bleaching product. ${ }^{12,13}$ Recently, two clinical studies showed that the application of calcium phosphate paste was effective in reducing at-home bleaching-induced tooth sensitivity. ${ }^{14,15}$ Grobler et al. ${ }^{14}$ hypothesized that the low sensitivity rate reported for an at-home bleaching gel was because of the presence of calcium phosphate, potassium nitrate, and fluoride in the gel composition.

A desensitizing paste composed of nano-calcium phosphate crystals with fluoride and potassium nitrate was recently introduced into the market (Nano-P, FGM, Joinville, Brazil). Although fluoride- and potassium nitrate-based desensitizing agents have been shown to reduce the risk of in-office bleaching-induced tooth sensitivity by half, ${ }^{9}$ no study has attempted to investigate the role of a nano-calcium phosphate paste associated with fluoride and potassium nitrate so far. According to the manufacturer, this product can obliterate dentinal tubules and remineralize the dental structure by deposition of stable nano-sized calcium phosphate.

Therefore, the present study aimed to evaluate bleaching-induced tooth sensitivity on application of a commercial nano-calcium phosphate paste containing fluoride and potassium nitrate prior to in-office bleaching. The following null hypotheses were tested: 1) the preventive use of a nano-calcium phosphate paste will not affect the absolute risk and intensity of bleaching-induced tooth sensitivity, and 2 ) the preventive use of a nano-calcium phosphate paste will not affect the color change after bleaching.

\section{Methodology}

This study was approved (protocol number 08272/2008) by the Ethics Committee of the Universidade Estadual de Ponta Grossa - UEPG (Paraná, Brazil). The study description follows the CONSORT statement. ${ }^{16}$ All volunteers underwent dental screening and dental prophylaxis two weeks prior to the bleaching procedures.

\section{Study design}

This was a randomized, parallel, double blinded, placebo-controlled trial with an equal allocation rate. The study was conducted at the School of Dentistry of the UEPG (Paraná, Brazil), between August 2009 and March 2010.

\section{Inclusion and exclusion criteria}

Participants included in this clinical trial were $\geq 18$ years old, and had good general and oral health. They were required to have six caries-free maxillary anterior teeth without restorations on the labial surfaces.

Central incisors were required to be shade $\mathrm{C}^{9,12,17}$ or darker in a value-oriented shade guide (Vita Lumin, Vita Zahnfabrik, Bad Säckingen, Germany). Exclusion criteria included previous tooth-whitening procedures, pregnancy/lactation, severe internal tooth discoloration (tetracycline stains, fluorosis, or pulpless teeth), any form of medication, bruxism, or any other pathology that could cause sensitivity (such as recession, dentin exposure). Participants that reported continuous use of anti-inflammatory drugs were also excluded from the study.

\section{Sample size calculation}

Thirty-eight patients (nineteen in each group) were required to have an $80 \%$ chance of detecting a decrease in the primary outcome measure from $83 \%$ (mean absolute risk of tooth sensitivity ${ }^{9,12,17}$ ) in the control group to $43 \%$ in the experimental group $(a=0.05)$. The sample size was calculated on the website www.sealedenvelope.com. The present study was powered to detect a high significant effect.

\section{Randomization, blinding and intervention}

The randomization process was performed by computer-generated tables. Details of the allocated 
group were recorded on cards contained in sequentially numbered, opaque, sealed envelopes. These were prepared by a staff member not involved in any of the phases of the clinical trial. The group allocation was revealed by opening the envelope on the day of the bleaching procedure. The operator was not blinded to group assignment when administering interventions, but participants and examiners (who assessed color) were.

Two clinicians applied either a placebo (carbopol-based gel mixed in water and thickener; Amanda, Farmácia de Manipulação, Ponta Grossa, Brazil) or the Nano-P paste (FGM) to the buccal tooth surfaces of all participants, and left it undisturbed for 10 minutes. The paste was then scrubbed using a rubber cup mounted on a slow-speed hand-piece on each tooth for 20 seconds. Although the placebo had the same packaging as the Nano-P, their consistency was different thus making it impossible to blind the operators.

The gingival tissues of the teeth were isolated using a light-polymerized resin dam (Top Dam, FGM). The 35\% hydrogen peroxide gel (Whiteness HP Maxx, FGM) was used in three 15 min applications in both groups. The in-office bleaching agent was refreshed every $15 \mathrm{~min}$ for a 45 -min period. Two bleaching sessions were performed, with an interval of one week. All participants were instructed to brush their teeth regularly using fluoridated toothpaste (Sorriso Fresh, Colgate-Palmolive, São Paulo, Brazil).

\section{Shade evaluation}

Shade evaluation was performed before and 1 week after the first and second bleaching sessions using the same shade guide described earlier. The shade tabs were organized from the whitest to the darkest, as follows: B1, A1, B2, D2, A2, C1, C2, D4, A3, D3, B3, A3.5, B4, C3, A4, and C4. For the purpose of analysis, we assumed that the color changes represented a continuous and approximately linear ranking. The shade matching was performed in the middle one third of the facial surface of the anterior central incisor. ${ }^{18,19}$

Two experienced and calibrated examiners, blinded to the group allocation, scheduled these participants for bleaching and evaluated their teeth against the shade guide at baseline, 1-week after the first appointment, and 1-week after the second appointment. The examiners were required to have an agreement of at least $85 \%$ (Kappa statistic) before examination.

\section{Tooth sensitivity evaluation}

The participants recorded their perception of tooth sensitivity during the first and second bleaching sessions, and up to $48 \mathrm{~h}$ after each bleaching session using a five-point verbal rating scale $(0=$ none, $1=$ mild, $2=$ moderate, $3=$ considerable, and $4=$ severe) ${ }^{12,13}$ One of the operators explained to the participants that zero meant no sensitivity at all, and 4 meant the worst kind of pain ever experienced.

\section{Statistical analysis}

The analysis followed the intention-to-treat protocol, and involved all participants who were randomly assigned. ${ }^{16}$ The statistician was blinded to the groups.

The worst score obtained in the entire treatment period was considered for statistical purposes. The absolute risk of tooth sensitivity (primary outcome) was compared using the Fisher's Exact test $(\alpha=0.05)$. The secondary outcome was tooth sensitivity intensity, and it was compared using the Mann-Whitney U-test $(\alpha=0.05)$, while the color change was analyzed using two-way repeated measures ANOVA (groups vs. assessment time) and Tukey's test $(\alpha=0.05)$.

\section{Results}

A total of 123 participants were examined to check if they were eligible (Figure). The mean age (years) of the participants was similar in the two groups (placebo $24.3 \pm 5.9$, and Nano-P $22.3 \pm 4.7$ years). Males accounted for $50 \%$ and $60 \%$ of the participants from the placebo and experimental groups, respectively.

\section{Tooth sensitivity}

In the placebo group, $80 \%$ (95\%CI58-91) experienced tooth sensitivity at least once during the bleaching regimen. In the Nano-P group the tooth sensitivity was $85 \%$ (95\%CI 63-94). No significant difference in the risk of tooth sensitivity was observed between 


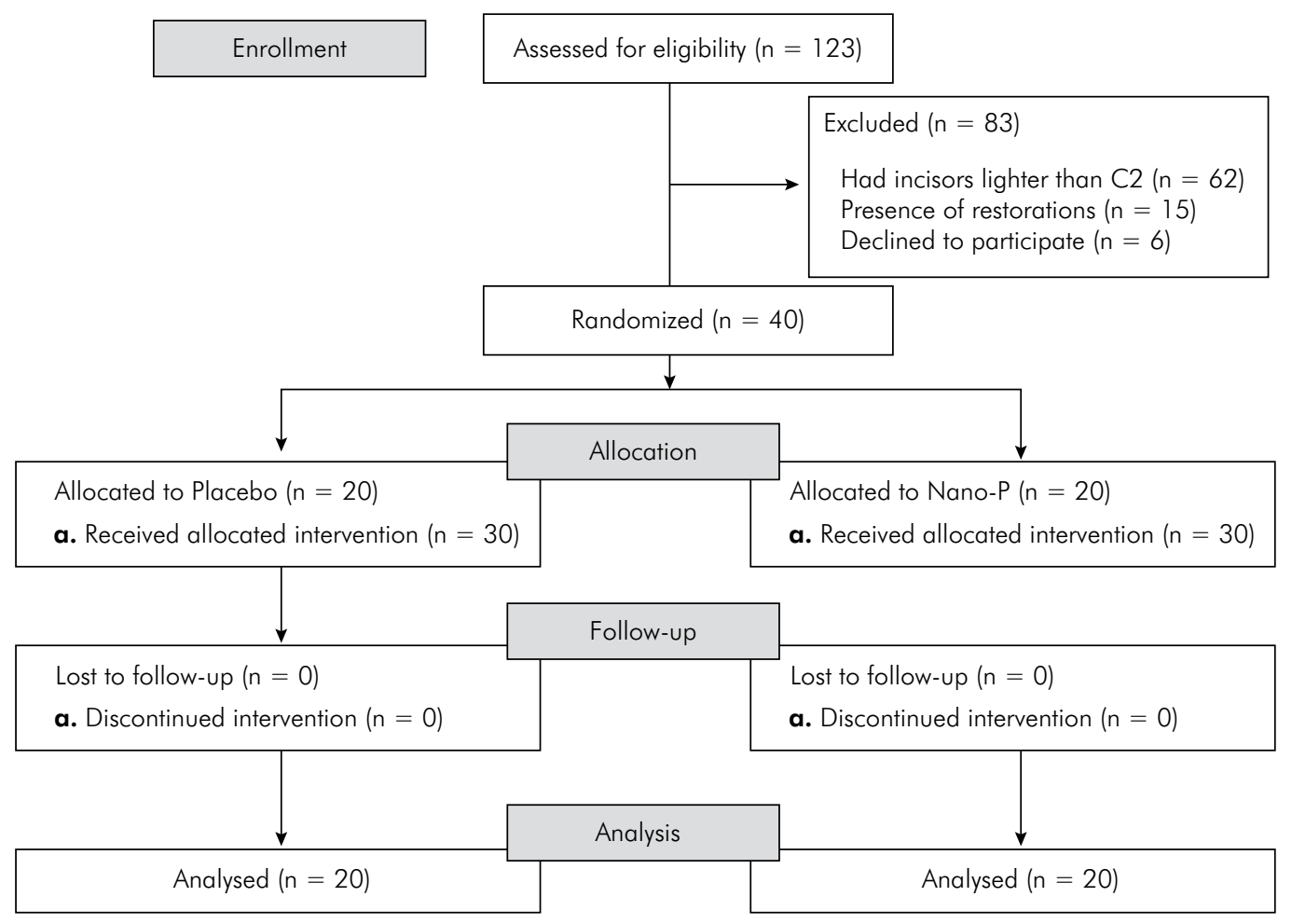

Figure. Flow-chart of patients.

the groups $(p=1.0)$. With regard to the intensity, the median of the placebo group was 2 ( 0 : minimum value, 3: maximum value), and the median of Nano-P group was 1.5 (0: minimum value, 3: maximum value). The groups did not differ statistically $(\mathrm{p}=0.53)$.

\section{Color evaluation}

Significant whitening was observed in both groups (Table, $\mathrm{p}<0.008$ ) but they did not differ from one another $(p=0.67)$. After two bleaching sessions, the mean color of the placebo and Nano-P groups were 1.5 (B1) and 2.1(A1), respectively.

\section{Discussion}

The absolute risk of tooth sensitivity reported in this study $(80 \%-85 \%)$ was similar to other papers reporting on the use of $35 \%$ hydrogen peroxide., ${ }^{9,12,17,20}$ Tooth sensitivity was not reduced by previous application of a nano-calcium phosphate paste; thus, preventing us from rejecting the first null hypothesis.

Although in vitro studies have reported changes in the enamel microhardness, ${ }^{4,5}$ when clinical conditions were simulated no alterations were seen
Table. Means (SD) of shade guide units at different times for experimental groups $\left({ }^{*}\right)$.

\begin{tabular}{lcc}
\hline \multicolumn{3}{c}{ Color evaluation-visual shade guide (Vita Classical) } \\
\hline Time assessment & \multicolumn{1}{c}{ Placebo } & Nano-P \\
\hline Baseline & $8.1 \pm 2.6 \mathrm{~A}$ & $7.7 \pm 2.3 \mathrm{~A}$ \\
1 week after first session & $4.2 \pm 2.1 \mathrm{~B}$ & $3.9 \pm 1.7 \mathrm{~B}$ \\
1 week after second session & $1.5 \pm 1.4 \mathrm{C}$ & $2.1 \pm 1.1 \mathrm{C}$ \\
\hline (*) Similar letters indicate statistically similar means (Two-way \\
ANOVA and the Tukey test, $\alpha=0.05$ ).
\end{tabular}

in the enamel microhardness, morphology, and calcium and phosphorus content associated with in-office bleaching. ${ }^{21,22}$ It appears that these small changes in the enamel produced by bleaching did not seem to be a problem as they were resolved by the remineralizing action of saliva. ${ }^{23}$ This explains why previous application of fluoride before bleaching was not capable of reducing the bleaching-induced tooth sensitivity. ${ }^{10}$

In addition to fluoride, the desensitizing paste used contained a nano-calcium phosphate compound which is believed to minimize reduction 
in enamel microhardness after bleaching by depositing calcium phosphate on the enamel surface, producing remineralization. In theory, calcium phosphates serve as a source of calcium and phosphate ions that interact with the substrate, thus increasing fluoride incorporation during the remineralization process which in turns reduces the solubility of enamel.

Based on the manufacturer's instructions, we hypothesized that Nano-P had a higher calcium/phosphate ratio. This assumption is based on the fact that the calcium phosphate from Nano-P is delivered in a crystalline form of hydroxyapatite under an alkaline $\mathrm{pH}^{24,25}$ which increases the availability of $\mathrm{Ca}^{+2}$ to interact with the substrate. ${ }^{26}$ However, in addition to the amount of available calcium, the contact time is also of paramount importance. This is why we applied the Nano-P paste for twice the amount of time recommended by the manufacturer.

The presence of $\mathrm{Ca}^{+2}$ is not the only factor that guarantees effective remineralization. ${ }^{24,25,26}$ The paste also needs to have a high solubility during application, and unfortunately, information on the solubility of different calcium phosphate compounds is still inaccurate in the literature. ${ }^{27}$

The exact composition of Nano-P paste is not known by the authors of this study, and not provided in detail by the manufacturer. Fernandez et al. ${ }^{24,25}$ indicated that several technological factors during manufacture and processing of these calcium phosphate compounds could affect their solubility parameters, which prevent us from making strong assumptions about this issue. As the paste application did not minimize bleaching-related tooth sensitivity, we assume that this commercial product has a low solubility to produce enamel remineralization.

Laboratory studies have shown that a paste containing nano-sized crystals can repair microscopic enamel pores. ${ }^{5,15}$ However, this appeared to be useless for the purpose of minimizing bleaching-induced tooth sensitivity. A recent in vitro study reported that the amount of hydrogen peroxide in enamel after application of the bleaching gel is minimal compared to dentin, ${ }^{28}$ suggesting that enamel, either in a sound, demineralized, or hypermineralized state, does not pose any barrier to penetration of the hydrogen peroxide.

Although this explains why the obliterating products (fluoride and calcium phosphates) did not reduce the tooth sensitivity, it does not seem to explain why the $5 \%$ potassium nitrate, added to the composition of the paste, was ineffective in reducing the tooth sensitivity. A recent study showed that a potassium nitrate/fluoride desensitizing agent applied before in-office bleaching reduced the tooth sensitivity by half, 9 a finding that was also reported by other studies that used potassium nitrate-based products along with bleaching therapy. ${ }^{8,11}$ Potassium nitrate penetrates into the enamel and dentin and reaches the pulp where it reduces the nerve depolarization and prevents the transmission of pain stimuli. $^{29}$

We hypothesized that a negative interaction between the calcium phosphate and potassium nitrate may have been responsible for this finding. Though the calcium phosphate salt deposited on the enamel surface is insoluble at an alkaline $\mathrm{pH}$, the bleaching gel used in this study is slightly acidic (approximately $5.5)^{6}$ which may have solubilized the phosphate salt. In solution, the phosphate ions become available for reaction with the soluble potassium, thereby reducing the availability of potassium ions to exert their calming effect on the pulp.

Unfortunately, there is a scarcity of literature on the effects of desensitizing pastes containing these three agents, and the results are still controversial. ${ }^{14,15}$ A recent study reported that the use of a nano-hydroxyapatite product in conjunction with at-home bleaching did not reduce the risk and intensity of tooth sensitivity. ${ }^{15}$ Other authors reported a low risk of bleaching-induced tooth sensitivity after at-home bleaching. ${ }^{14}$ This study, however, should be interpreted with caution since the authors did not add any control group in the experimental design. This reinforces the need for further studies on this matter.

With respect to color change, the results of this study indicate that preliminary application of the desensitizing agent with obliterating compounds did not jeopardize the whitening effect. An equivalent and significant tooth color enhancement was observed in both study groups. The degree of 
whitening obtained in this study is similar to that reported in other clinical trials ${ }^{9,17,19}$ and also in the literature review.

Finally, it should be mentioned that only a value-oriented shade guide was used for identification of color changes throughout the study period. This was done because it is an easy, fast, and satisfactory method used in several investigations. ${ }^{9,17,19}$ Additionally, a recent study showed that visual assessment of tooth color with a value-oriented shade guide is a valid method, with good reliability in differentiating between dark and light colors. ${ }^{30}$

\section{References}

1. Matis BA, Cochran MA, Eckert G. Review of the effectiveness of various tooth whitening systems. Oper Dent. 2009 Mar-Apr;34(2):230-5.

2. Haywood VB. Treating sensitivity during tooth whitening. Compend Contin Educ Dent. 2005 Sep;26(9):11-20.

3. Akal N, Over H, Olmez A, Bodur H. Effects of carbamide peroxide containing bleaching agents on the morphology and subsurface hardness of enamel. J Clin Pediatr Dent. 2001 Summer;25(4):293-6.

4. Cavalli V, Rodrigues LK, Paes-Leme AF, Brancalion ML, Arruda MA, Berger SB, et al. Effects of bleaching agents containing fluoride and calcium on human enamel. Quintessence Int. 2010 Sep;41(8):157-65.

5. Soares MUC, Araújo NC, Borges BC, Sales WS, Sobral AP. Impact of remineralizing agents on enamel microhardness recovery after in-office tooth bleaching therapies. Acta Odontol Scand. 2013 Mar;71(2):343-8.

6. Freire A, Archegas LR, Souza EM, Vieira S. Effect of storage temperature on $\mathrm{pH}$ of in-office and at-home dental bleaching agents. Acta Odontol Latinoam. 2009;22(1):27-31.

7. Goldberg M, Grootveld M, Lynch E. Undesirable and adverse effects of tooth-whitening products: a review. Clin Oral Investig. 2010 Feb;14(1):1-10.

8. Kose C, Reis A, Baratieri LN, Loguercio AD. Clinical effects of at-home bleaching along with desensitizing agent application. Am J Dent. 2011 Dec;24(6):379-82.

9. Tay LY, Kose C, Loguercio AD, Reis A. Assessing the effect of a desensitizing agent used before in-office tooth bleaching J Am Dent Assoc. 2009 Oct;140(10):1245-51.

10. Armênio RV, Fitarelli F, Armênio MF, Demarco FF, Reis A, Loguercio AD. The effect of fluoride gel use on bleaching sensitivity: a double-blind randomized controlled clinical trial. J Am Dent Assoc. 2008 May;139(5):592-7.

\section{Conclusion}

The use of a nano-calcium phosphate paste containing potassium nitrate, fluoride, and calcium phosphate prior to in-office bleaching did not reduce bleaching-induced tooth sensitivity measured during and up to $48 \mathrm{~h}$ after each session.

\section{Acknowledgements}

This study was partially supported by the Fundação de Amparo à Pesquisa e Desenvolvimento Científico do Maranhão - FAPEMA and by the Conselho Nacional de Desenvolvimento Científico e Tecnológico (CNPq) under grants 304104/2013-9 and 301891/2010-9.

11. Leonard RH Jr., Smith LR, Garland GE, Caplan DJ. Desensitizing agent efficacy during whitening in an at-risk population. J Esthet Restor Dent. 2004;16(1):49-55.

12. Kossatz S, Martins G, Loguercio AD, Reis A. Tooth sensitivity and bleaching effectiveness associated with use of a calcium-containing in-office bleaching gel. J Am Dent Assoc. 2012 Dec;143(12):81-7.

13. Giniger M, Spaid M, MacDonald J, Felix H. A 180-day clinical investigation of the tooth whitening efficacy of a bleaching gel with added amorphous calcium phosphate. J Clin Dent. 2005;16(1):11-6.

14. Grobler SR, Majeed A, Moola MH, Rossouw RJ, van Wyk Kotze T. In vivo spectrophotometric assessment of the tooth whitening effectiveness of Nite White $10 \%$ with amorphous calcium phosphate, potassium nitrate and fluoride, over a 6-month period. Open Dent J. 2011 Mar 2;5:18-23.

15. Browning WD, Cho SD, Deschepper EJ. Effect of a nano-hydroxyapatite paste on bleaching-related tooth sensitivity. J Esthet Restor Dent. 2012 Aug;24(4):268-76.

16. Schulz KF, Altman DG, Moher D. CONSORT 2010 statement: updated guidelines for reporting parallel group randomised trials. PLoS Med. 2010 Mar 24;7(3):e1000251.

17. Reis A, Tay LY, Herrera DR, Kossatz S, Loguercio AD. Clinical effects of prolonged application time of an in-office bleaching gel. Oper Dent. 2011 Nov-Dec;36(6):590-6.

18. Cardoso PC, Reis A, Loguercio A, Vieira LC, Baratieri LN. Clinical effectiveness and tooth sensitivity associated with different bleaching times for a 10 percent carbamide peroxide gel. J Am Dent Assoc. 2010 Oct;141(10):1213-20.

19. Marson FC, Sensi LG, Vieira LC, Araujo E. Clinical evaluation of in-office dental bleaching treatments with and without the use of light-activation sources. Oper Dent. 2008 Jan-Feb;33(1):15-22. 
20. Alomari Q, El Daraa E. A randomized clinical trial of in-office dental bleaching with or without light activation. J Contemp Dent Pract. 2010 Jan 1;11(1):E017-24.

21. Cadenaro M, Breschi L, Nucci C, Antoniolli F, Visintini E, Prati C, et al. Effect of two in-office whitening agents on the enamel surface in vivo: a morphological and non-contact profilometric study. Oper Dent. 2008 Mar-Apr;33(2):127-34.

22. Amaral FL, Sasaki RT, Silva TC, França FM, Flório FM, Basting RT. The effects of home-use and in-office bleaching treatments on calcium and phosphorus concentrations in tooth enamel: an in vivo study. J Am Dent Assoc. 2012 Jun;143(6):580-6.

23. Attin T, Schmidlin PR, Wegehaupt F, Wiegand A. Influence of study design on the impact of bleaching agents on dental enamel microhardness: a review. Dent Mater. 2009 Feb;25(2):143-57.

24. Fernández E, Gil FJ, Ginebra MP, Driessens FC, Planell JA, Best SM. Calcium phosphate bone cements for clinical applications. Part I: solution chemistry. J Mater Sci Mater Med. 1999 Mar;10(3):169-76.
25. Fernández E, Gil FJ, Ginebra MP, Driessens FC, Planell JA, Best SM. Calcium phosphate bone cements for clinical applications. Part II: precipitate formation during setting reactions. J Mater Sci Mater Med. 1999 Mar;10(3):177-83.

26. Liu Q, Huang S, Matinlinna JP, Chen Z, Pan H. Insight into biological apatite: physiochemical properties and preparation approaches. Biomed Res Int. 2013 Sep;2013:929748.

27. Horvath L. Solubility of structurally complicated materials: II. Bone. J Phys Chem Ref Data. 2006 Nov;35(4):1653-68.

28. Ubaldini AL, Baesso ML, Medina Neto A, Sato F, Bento AC, Pascotto RC. Hydrogen peroxide diffusion dynamics in dental tissues. J Dent Res. 2013 Jul;92(7):661-5.

29. Ajcharanukul O, Kraivaphan P, Wanachantararak S, Vongsavan N, Matthews B. Effects of potassium ions on dentine sensitivity in man. Arch Oral Biol. 2007 Jul;52(7):632-9.

30. Meireles SS, Heckmann SS, Leida FL, Santos IS, Della Bona A, Demarco FF. Efficacy and safety of $10 \%$ and $16 \%$ carbamide peroxide tooth-whitening gels: a randomized clinical trial. Oper Dent. 2008 Nov-Dec;33(6):606-12. 